\title{
Marine Plastics from Norwegian West Coast Carry Potentially Virulent Fish Pathogens and Opportunistic Human Pathogens Harboring New Variants of Antibiotic Resistance Genes
}

\author{
Vera Radisic ${ }^{1,2}$, Priyank S. Nimje ${ }^{1}$, André Marcel Bienfait ${ }^{1}$ and Nachiket P. Marathe ${ }^{1, *(D)}$ \\ 1 Institute of Marine Research, 5005 Bergen, Norway; veraradisic96@gmail.com (V.R.); \\ priyankn21@gmail.com (P.S.N.); andre.marcel.bienfait@hi.no (A.M.B.) \\ 2 Department of Biological Sciences, University of Bergen, 5006 Bergen, Norway \\ * Correspondence: nachiket.marathe@hi.no
}

Received: 9 July 2020; Accepted: 4 August 2020; Published: 7 August 2020

\begin{abstract}
To our best knowledge this is the first study characterizing fish pathogens isolated from marine plastics from the West coast of Norway for their potential for pathogenicity using whole genome sequencing. Marine plastic polymers identified as polyethylene, polyethylene/ethylene vinyl acetate copolymer and polypropylene, yielded a total of 37 bacterial isolates dominated by Pseudomonas spp. $(70 \%)$. Six isolates representing either fish pathogens or opportunistic human pathogens were selected for whole genome sequencing (WGS). These included four isolates belonging to Aeromonas spp., one Acinetobacter beijerinckii isolate and one Morganella morganii isolate. Three Aeromonas salmonicida isolates were potentially virulent and carried virulence factors involved in attachment, type II and type VI secretion systems as well as toxins such as aerA/act, ahh1, ast, hlyA, rtxA and toxA. A. salmonicida and Acinetobacter beijerinckii carried new variants of antibiotic resistance genes (ARGs) such as $\beta$-lactamases and chloramphenicol acetyltransferase (catB), whereas Morganella morganii carried several clinically relevant ARGs. Our study shows that marine plastics carry not only potentially virulent fish pathogens but also multidrug resistant opportunistic human pathogens like M. morganii and may serve as vectors for transport of these pathogens in the marine environment.
\end{abstract}

Keywords: marine plastics; microplastics; fish pathogens; antibiotic resistance; Norway; Aeromonas sp.

\section{Introduction}

Plastics are synthetic, anthropogenic materials made from a wide range of organic polymers such as polyethylene (PE), polypropylene (PP), polystyrene (PS), polyvinyl chloride (PVC), polyurethane (PUR), polyethylene terephthalate (PET), polybutylene terephthalate (PBT) and polyamide (PA), commonly known as nylon [1]. Plastic materials enter the marine ecosystem in many different ways, such as being dumped, lost [2], carelessly handled [2,3] or left [2,4]. Plastics found in the environment are mostly classified into three different categories based on their sizes: nanoplastic $(<100 \mathrm{~nm})$, microplastic $(100 \mathrm{~nm}-5 \mathrm{~mm})$ and macroplastic $(>5 \mathrm{~mm})$ [5-7].

Pollution with plastics and accumulation of plastics in the oceans has a big impact on the marine ecosystem [8-10]. Ingestion of plastic by marine organisms can disturb their energy balance, affect behavior and sometimes block the intestinal tract leading to sublethal effect or, in the worst-case, cause death [11,12]. Small microplastic $(<10 \mu \mathrm{m})$ and nanoplastic particles appear to have an increased plastic particle toxicity to aquatic life [13]. Plastic can also be toxic due to the breakdown products as well as due to the presence of chemicals absorbed or released from plastics, such as plasticizers [14-16]. 
Although some polymers are biodegradable, albeit at a very slow rate, others are not [1] and thus plastics stay in the environment for a long time.

Plastic provides a hydrophobic surface for the attachment of microbes and hence different microbes colonize plastics in the marine environment $[5,17,18]$. Therefore, marine plastics may function as vectors for the transport of bacteria, including fish pathogens, in the oceans [19]. Colonization of plastics by bacteria can also facilitate horizontal gene transfer of antibiotic resistance genes (ARGs), owing to biofilm formation [20]. Accordingly, a study has shown presence of multidrug resistant bacteria on plastics from marine environments [5].

Plastic pollution has been reported in the marine environment in Norway [21]. To date, Norway is a country with a low burden of antibiotic resistance in clinics and in the environment [22]. Although this is true, there is lack of knowledge about microbiota associated with plastic found in the marine environment in Norway as well as associated ARGs. The aim of our study was to investigate the presence of fish pathogens and antibiotic resistant bacteria on marine plastics collected from the West coast of Norway. We isolated fish pathogens and opportunistic human pathogens from plastic surfaces, analyzed whole genome sequences of these isolates and report their potential for pathogenicity as well as new variants of ARGs.

\section{Materials and Methods}

\subsection{Sample Collection}

Macroplastic samples $(n=7)$, with different sizes ranging from one centimeter to $15 \mathrm{~cm}$, were collected from the intertidal zone at beaches in Øygarden, Vestland county, Norway. The coordinates for the sampling areas are $60^{\circ} 29^{\prime} 57.6^{\prime \prime} \mathrm{N} 4^{\circ} 55^{\prime} 05.3^{\prime \prime} \mathrm{E}$ and $60^{\circ} 29^{\prime} 54.5^{\prime \prime} \mathrm{N} 4^{\circ} 54^{\prime} 52.6^{\prime \prime} \mathrm{E}$, respectively. The samples were collected in either sterile $50 \mathrm{~mL}$ tubes or sterile plastic bags. These samples were then stored at $4{ }^{\circ} \mathrm{C}$ and transported back to the lab for analysis.

\subsection{Isolation and Identification of Bacterial Strains}

Plastic samples were carefully washed with sterile phosphate buffer saline (PBS) before making suspensions. Suspensions were made by adding $10 \mathrm{~mL}$ of sterile PBS to each plastic sample in $50 \mathrm{~mL}$ sterile tubes. Four sterile glass beads ( $4 \mathrm{~mm}$ diameter) were added to the tubes, followed by vortexing for $90 \mathrm{~s}$ at maximum speed. Serial dilutions of the suspension were prepared in sterile PBS and spread on Mueller-Hinton agar plates and MacConkey agar plates, both media containing either ampicillin $(100 \mu \mathrm{g} / \mathrm{mL})$, cefotaxime $(10 \mu \mathrm{g} / \mathrm{mL})$ or no antibiotics. Mueller-Hinton agar plates were incubated at $25^{\circ} \mathrm{C}$ for $24-48 \mathrm{~h}$, while MacConkey agar plates were incubated at $37^{\circ} \mathrm{C}$ for $24-48 \mathrm{~h}$. A total of 37 colonies were picked and purified by restreaking on Mueller-Hinton agar with ampicillin. Fish pathogens like Aeromonas spp. and Vibrio spp. are intrinsically resistant to penicillins, thus, ampicillin facilitated selection of fish pathogens [23,24]. All isolates were stored as glycerol stocks at $-80{ }^{\circ} \mathrm{C}$ for further use. For identification, isolates were grown on Mueller-Hinton agar with ampicillin at $30{ }^{\circ} \mathrm{C}$ for $24 \mathrm{~h}$. The strains were typed using a Bruker MALDI Biotyper at Veterinær instituttet (Bergen, Norway) using the MALDI Biotyper database.

\subsection{Genomic DNA Extraction and Illumina Sequencing}

Genomic DNA was extracted from isolates cultivated overnight on Mueller-Hinton agar with ampicillin using QlAamp Fast DNA Stool Mini Kit (Qiagen, Hilden, Germany) following the manufacturer's instructions. NanoDrop 1000 and Qubit 2.0 (Thermo Scientific, Waltham, MA, USA) were used to quantify the extracted DNA. Extracted DNA was sent for sequencing to the Norwegian Sequencing Centre (Oslo University Hospital, Ullevål, Oslo, Norway). Nextera DNA Flex Library Prep Kit (Illumina, San Diego, CA, USA) was used for preparing sequencing libraries. Sequencing was performed on an Illumina MiSeq platform (Illumina, San Diego, CA, USA), using $2 \times 300$ bp chemistry. 


\subsection{Genome Sequence Assembly and Screening for ARGs}

Adapters were removed from the obtained raw reads and the reads were quality filtered using BBDuk (version 38.75; https://jgi.doe.gov/data-and-tools/bbtools/bb-tools-user-guide/). Sequences were assembled in SPAdes (version 3.13.0) [25] using the default parameters; spades.py -1 xx_R 1 _trim.fastq -2 xx_R 2 _trim.fastq -careful -only-assembler -cov-cutoff auto -o output_name. Genome annotation was performed using National Center for Biotechnology Information (NCBI) Prokaryotic Genomes Automatic Annotation Pipeline (PGAAP) [26]. The genome sequences were screened for antibiotic resistance genes using the CARD database (version 3.0.7) [27] and ResFinder 3.2 database [28]. The virulence genes were identified using VFanalyzer and the virulence factor database (VFDB) [29]. Genome sequences have been submitted to GenBank under the following genome accession numbers: JAACGC000000000, JAACGB000000000, JAACGA000000000, JAACFZ000000000, JAACFY000000000, JAACFX000000000 and JAACFW000000000, respectively.

\subsection{Phylogenetic Analysis}

Amino acid sequences for CphA, RtxA and AerA were extracted from the genome sequences of Aeromonas spp. Additional sequences for respective proteins were downloaded from GenBank (www.ncbi.nlm.nih.gov/genbank). The sequences were aligned using clustalX version 2.1 [30]. The phylogenetic tree was generated by the neighbor joining method using MEGA-X with bootstrap analysis (1000 replicates) [31].

\subsection{Antibiotic Susceptibility Testing}

Isolates were grown on Mueller-Hinton agar with ampicillin at $30^{\circ} \mathrm{C}$ overnight and were used for making suspensions for determination of minimum inhibitory concentration (MIC). MICs were determined for cefotaxime (CT), tetracycline (TC), ciprofloxacin (CI), ampicillin (AM), meropenem (MP), streptomycin (SM), trimethoprim (TR), gentamicin (GM), imipenem (IP) and chloramphenicol (CL) using E-test according to manufacturer's protocol (bioMérieux, Paris, France). Escherichia coli strain CCUG 17,620 was used as a control strain for E-test quality check.

\subsection{Identifying Plastic Polymer Type}

The polymer type for plastic samples was identified by attenuated total reflection infrared spectroscopy (Cary670 FTIR spectrometer, Agilent Technologies) equipped with a monolithic diamond crystal unit (GladiATR ${ }^{\mathrm{TM}}$, PIKE Technologies, Madison, WI, USA). Spectra were collected over the wavenumber range $440-4000 \mathrm{~cm}^{-1}$. The samples were analyzed by 32 co-added scans, with a resolution of $8 \mathrm{~cm}^{-1}$. The spectra were compared to spectra of known standards using a library of environmental relevant synthetic and natural polymers [32], and commercial libraries for polymers (Bio-Rad Sadtler) in the KnowitAll Informatics System software (BioRad Laboratories, Hercules, CA, USA). Each polymer type was determined by a combination of the best-fitted spectra and the expertise of the operator on interpreting polymer IR spectra.

\section{Results}

\subsection{Identification of the Plastic Polymers and Bacterial Strains}

The plastic polymers were identified as polyethylene, polyethylene/ethylene vinyl acetate copolymer or polypropylene (Figure 1). A total of 37 isolates were obtained from the samples (Table S1). Only two isolates were obtained from polypropylene, while the rest were found on polyethylene or polyethylene/ethylene vinyl acetate copolymer. Pseudomonas spp. dominated the isolates (26 of 37). Four isolates representing fish pathogens Aeromonas spp. and two isolates representing opportunistic human pathogens (Morganella morganii and Acinetobacter beijerinckii, respectively) were chosen for characterization with whole genome sequencing (WGS). Genome sequence assembly statistics and 
GenBank accession number for the genome sequences of the isolates are represented in Table S2. Sequencing coverage ranged from $55 \times$ to $110 \times$.

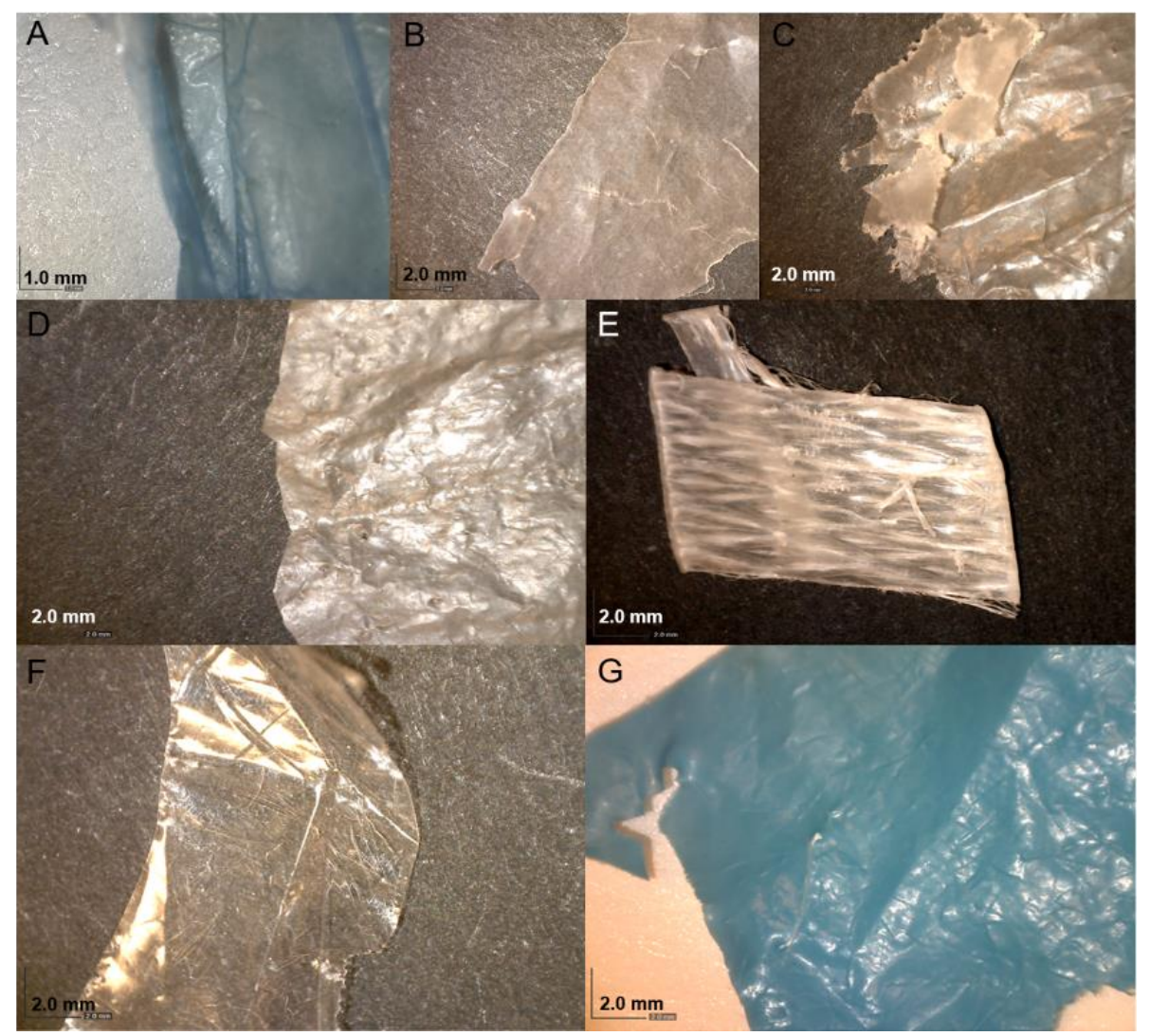

Figure 1. Images of samples collected in this study. (A,C,D,F,G) are polyethylene; (B) is polyethylene/ethylene vinyl acetate copolymer; $(\mathbf{E}, \mathbf{F})$ are polypropylene.

\subsection{Antibiotic Resistance and ARGs}

All three A. salmonicida isolates were resistant to ampicillin. M. morganii and Ac. beijerinckii were multidrug resistant, with resistance against at least three different classes of antibiotics (Table 1). Class C $\beta$-lactamases and chloramphenicol acetyltransferase (cat $B)$ were detected in all six isolates. Class B2 metallo- $\beta$-lactamase $c p h A$ was detected in three $A$. salmonicida isolates, with a new variant of $c p h A$ ( $\leq 98 \%$ identity) present in two of the three $A$. salmonicida isolates (Table 2 and Figure S1). Along with cphA variants all Aeromonas isolates carried qnrA gene. Isolate $2 \mathrm{HC} 4$ (Ac. beijerinckii) carried new variants of four different ARGs including a class A and a class $C \beta$-lactamase, aminoglycoside acetyltransferase and chloramphenicol acetyltransferase. M. morganii carried several resistance genes with $100 \%$ homology to previously described ARGs. The list of ARGs detected in the genome sequences of these isolates is presented in Table 2.

\subsection{Virulence Factors}

All three $A$. salmonicida isolates had genes for virulence factors involved in attachment, type II (T2SS) and type VI (T6SS) secretion systems as well as toxins such as aerA/act, ahh1, ast, hlyA, rtxA, rtxB, $r t x C, r t x D, r t x E, r t x H$ and toxA. This suggests that they have potential for causing infection. M. morganii carried genes involved in acid resistance, genes involved in iron uptake, and type six secretion system proteins. A phylogenetic tree of RtxA and AerA proteins from A. salmonicida isolates and closely related proteins from other species is presented in Figure 2. A list of virulence genes detected in all sequenced isolates is presented Table S3. 
Table 1. Minimum inhibitory concentration (MIC) of different antibiotics.

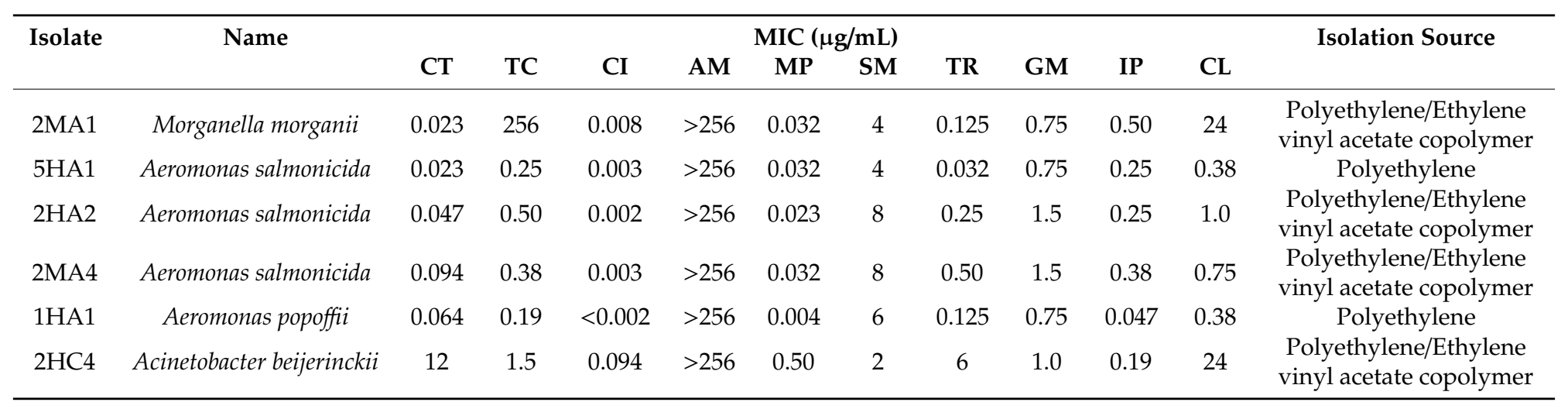

Legend: CT (cefotaxime), TC (tetracycline), CI (ciprofloxacin), AM (ampicillin), MP (meropenem), SM (streptomycin), TR (trimethoprim), GM (gentamicin), IP (imipenem) and CL (chloramphenicol)

Table 2. Overview of the different antibiotic resistance genes (ARGs) detected in whole genome sequence of the isolates.

\begin{tabular}{|c|c|c|c|c|}
\hline Isolate & Name & Gene & Closest Blast Hit & Percent Identity (Amino Acid) \\
\hline \multirow[t]{4}{*}{ 2MA1 } & Morganella morganii & $b l a_{\mathrm{DHA}}$ & DHA family class $C \beta$-lactamase & $100.00 \%$ \\
\hline & & $\operatorname{tet}(D)$ & tetracycline efflux MFS transporter Tet(D) & $100.00 \%$ \\
\hline & & $\operatorname{aac}(3)$ & aminoglycoside 3-N-acetyltransferase & $100.00 \%$ \\
\hline & & $\operatorname{cat} B$ & antibiotic acetyltransferase & $100.00 \%$ \\
\hline \multirow{5}{*}{$5 \mathrm{HA} 1$} & Aeromonas salmonicida & $\operatorname{ampC}$ & FOX/MOX family class $C \beta$-lactamase & $98.43 \%$ \\
\hline & & $b l a_{\mathrm{OXA}}$ & class $\mathrm{D} \beta$-lactamase & $100.00 \%$ \\
\hline & & $\operatorname{cph} A$ & CphA family subclass B2 metallo- $\beta$-lactamase & $97.64 \%$ \\
\hline & & $q n r A$ & Qnr family pentapeptide repeat protein & $100.00 \%$ \\
\hline & & catB & antibiotic acetyltransferase & $98.64 \%$ \\
\hline \multirow[t]{5}{*}{ 2HA2 } & Aeromonas salmonicida & $\operatorname{ampC}$ & FOX/MOX family class $C \beta$-lactamase & $97.90 \%$ \\
\hline & & $\operatorname{cph} A$ & CphA family subclass B2 metallo- $\beta$-lactamase & $99.61 \%$ \\
\hline & & $b l a_{\mathrm{OXA}}$ & class $\mathrm{D} \beta$-lactamase & $100.00 \%$ \\
\hline & & $q n r A$ & Qnr family pentapeptide repeat protein & $100.00 \%$ \\
\hline & & catB & antibiotic acetyltransferase & $98.64 \%$ \\
\hline
\end{tabular}


Table 2. Cont

\begin{tabular}{|c|c|c|c|c|}
\hline Isolate & Name & Gene & Closest Blast Hit & Percent Identity (Amino Acid) \\
\hline \multirow[t]{5}{*}{ 2MA4 } & Aeromonas salmonicida & $\operatorname{ampC}$ & FOX/MOX family class $C \beta$-lactamase & $96.33 \%$ \\
\hline & & $b l a_{\mathrm{OXA}}$ & class D $\beta$-lactamase & $99.24 \%$ \\
\hline & & cphA & CphA family subclass B2 metallo- $\beta$-lactamase & $96.85 \%$ \\
\hline & & $q n r A$ & Qnr family pentapeptide repeat protein & $100.00 \%$ \\
\hline & & cat $B$ & antibiotic acetyltransferase & $99.09 \%$ \\
\hline \multirow[t]{4}{*}{ 1HA1 } & Aeromonas popoffii & $b l a_{\mathrm{OXA}}$ & class $\mathrm{D} \beta$-lactamase & $99.62 \%$ \\
\hline & & $\operatorname{ampC}$ & FOX/MOX family class $C \beta$-lactamase & $98.95 \%$ \\
\hline & & $q n r A$ & Qnr family pentapeptide repeat protein & $100.00 \%$ \\
\hline & & catB & antibiotic acetyltransferase & $99.10 \%$ \\
\hline \multirow[t]{4}{*}{$2 \mathrm{HC} 4$} & Acinetobacter beijerinckii & $\operatorname{ampC}$ & class $C \beta$-lactamase & $96.46 \%$ \\
\hline & & bla & class A $\beta$-lactamase & $96.11 \%$ \\
\hline & & $\operatorname{aac}\left(6^{\prime}\right)-I$ & AAC $\left(6^{\prime}\right)$-Ighjkrstuvwx family aminoglycoside $\mathrm{N}$-acetyltransferase & $97.93 \%$ \\
\hline & & catB & antibiotic acetyltransferase & $96.24 \%$ \\
\hline
\end{tabular}



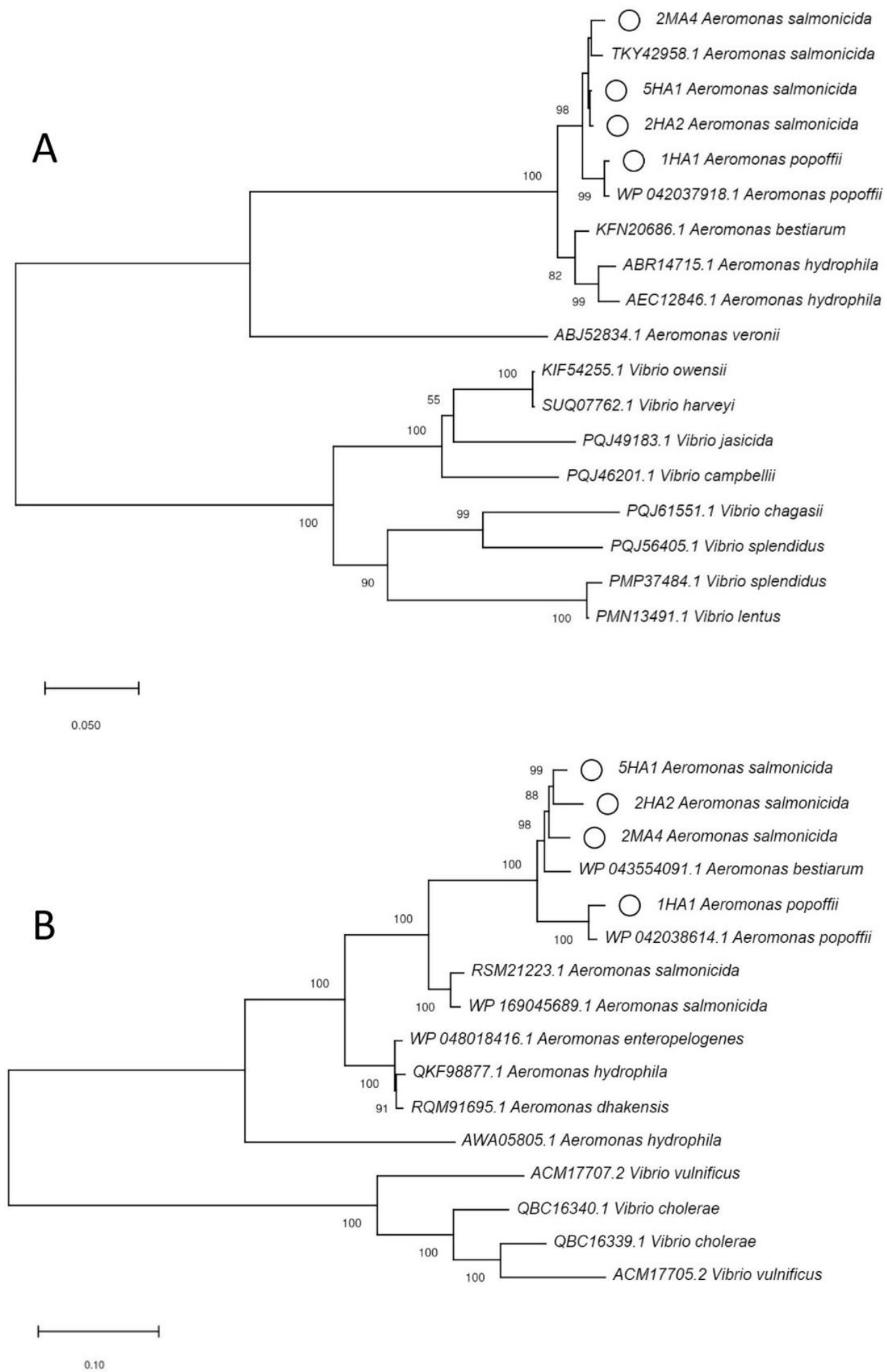

Figure 2. (A) Phylogenetic tree of AerA based on amino acid sequences. (B) Phylogenetic tree of RtxA based on amino acid sequences. The proteins highlighted with small circles are found in A. salmonicida isolates in this study. Accession numbers and host organisms are presented for sequences downloaded from GenBank. Numbers at nodes are bootstrap values (\%) based on 1000 resampled datasets; only values $>50 \%$ are given. 


\section{Discussion}

To our best knowledge, this is the first study analyzing genome sequences of multidrug resistant bacteria, including fish pathogens, associated with plastic found in the marine environment in Norway. Using 16S rDNA clone libraries, Viršek et al. [33] suggested that microplastics might serve as a vector for the transport of pathogenic bacteria, especially fish pathogen A. salmonicida. Our study strengthens the notion that fish pathogens as well as other opportunistic human pathogens are present on marine plastics. We further show the presence of virulence genes and new variants of antibiotic resistance genes in A. salmonicida isolated from these plastics.

Most Aeromonas spp. are opportunistic pathogens and are widespread in the marine environments [34,35]. Aeromonas spp. are known to cause infection in both humans and animals [36]. A. salmonicida is one of the most important fish pathogens [37], causing disease in healthy wild and cultured stocks of salmon and other fish species [36]. All A. salmonicida isolates in this study carried virulence factors like the $r t x A$ toxin. Repeats in toxins (RTX) $r t x A$ is a cytotoxin that is an important virulence factor for many fish pathogens, including Vibrio spp. and Aeromonas spp. It disrupts host cells membranes, aiding pathogenesis [38,39]. Along with $r t x A$, other cytotoxins like aerolysin aer $A$ [40], extracellular hemolysin ahh1, heat-stable cytotonic enterotoxin ast, hemolysin III and hylA were detected in these isolates [41]. A study suggests that the presence of both aerA and ahh1 represents the most cytotoxic genotype in A. hydrophila [41]. In addition, Aeromonas isolates also carried Exotoxin-A (toxA), which is a major virulence factor in Pseudomonas aeruginosa [42]. Two A. salmonicida isolates (2HA2 and 2MA4) carried genes involved in immune evasion (capsular polysaccharide), phagocytosis prevention $(r m l C, w b f U, w b f Y)$ and endotoxin from Bordetella. In addition to these, several others virulence factors like adhesion factors, T2SS $[43,44]$ and T6SS $[45,46]$ were detected. The presence of virulence factors in the genome does not necessarily guarantee expression of these virulence factors and further in-depth studies are needed to establish the virulence of these isolates. Nevertheless, the presence of these virulence genes indicates that these isolates have potential for causing infections. This emphasizes the risk posed by plastics as vectors for the transport of potentially virulent fish pathogens in the marine environment, especially in Norway where aquaculture is one of the major activities.

All three isolates of $A$. salmonicida in our study carried resistance genes like $\operatorname{amp} C$, bla $a_{\mathrm{OXA}}, c p h A$, $q n r A$ and cat $B$. New variants of $c p h A$ (Class B2 metallo- $\beta$-lactamase) were present in the two isolates (5HA1 and 2MA4) of $A$. salmonicida whereas new variants of FOX/MOX family class $C \beta$-lactamase (96.3\% to $98.4 \%$ identity) were detected in all three isolates. Marine bacteria have been shown to be a source of ARGs found in the clinics [47-49]. A recent study by Ebmeyer et al. [50] showed that Aeromonas spp. are the origin of several clinically important $\beta$-lactamases like CMY-1/MOX-family AmpC $\beta$-lactamases MOX-1, MOX-2 and MOX-9. Similarly, another study from Ebmeyer et al. [51] showed that FOX AmpC $\beta$-lactamases originated in A. allosaccharophila. They proposed that the mobilization and fixation of these genes may be recent and may have happened during the antibiotic era. Plastics provide a hydrophobic surface for the attachment of microbes promoting colonization and biofilm formation [52]. In accordance, an increased frequency of resistance plasmid transfer in bacteria associated with microplastics was observed [20]. Plastics also absorb a range of pollutants including antibiotic and heavy metals that are known to be drivers of antibiotic resistance [5,53-55]. This may create selection pressure, aiding transfer and/or mobilization of ARGs in the surface associated microbiota on marine plastics [56]. In order to better understand the role of marine plastics in mobilization and selection of ARGs, more research is thus warranted.

We detected opportunistic human pathogens like M. morganii [57-59], Ac. beijerinckii [60] and A. popoffii [61] on plastic surfaces. M. morganii previously belonged to family Enterobacteriaceae, which also consists of classical human pathogens like Escherichia coli, Salmonella typhi and Klebsiella pneumoniae. It was recently reclassified to be included in its own family Morganellaceae [62]. Although reclassified, $M$. morganii is an important emerging opportunistic human pathogen causing a variety of infections ranging from wound infections, urinary tract infections to meningitis [57-59]. M. morganii isolate (2MA1) carried virulence genes involved in acid resistance (ureB, ure G), genes 
involved in iron uptake, and T6SS proteins. Further, this isolate was resistant to tetracycline, chloramphenicol and $\beta$-lactams as well as carried several clinically important resistance genes like tet $D, a a c 3$ and $c a t B$ [59]. The presence of multi-drug resistant human associated bacteria carrying multiple ARGs suggests that plastic may serve as vectors for transport of not only fish pathogens but also opportunistic human pathogens in the marine environment. Our results are in accordance with a recent study that showed presence of potentially pathogenic bacteria on plastic debris from Guanabara Bay in Brazil [63].

Aquaculture is both historically and economically important for Norway. Norway is considered the world's second largest exporter of seafood after China and delivers fish to more than 100 countries [64,65]. In 2018, more than 1.35 million ton of fish, mostly salmon (Salmo salar) (1.28 million ton) and rainbow trout (Oncorhynchus mykiss) (68,345 ton) were farmed in Norway, with a first-hand value of 67.8 billion NOK (8.34 billion USD) $[66,67]$. The presence and spread of potentially virulent fish pathogens on marine plastics, could have a major impact on aquaculture in Norway. Hence, more research on understanding of the role of plastic surface-associated microbial communities and their biogeochemical functions in the marine environment is needed, especially in Norway.

\section{Conclusions}

Our study demonstrates the presence of fish pathogens and human associated bacteria on marine plastics from Norway. We show the potential for pathogenicity of $A$. salmonicida isolates obtained from marine plastics, with presence of genes encoding toxins, hemolysins and adhesion factors in their genome sequences, as well as describe new variants of ARGs carried by plastic associated bacteria. Our study strengthens the notion that plastic debris may serve as vectors for transport for fish pathogen as well as other opportunistic human pathogens in the marine environment. Marine plastics colonized by potentially virulent fish pathogens may impact aquaculture. Hence, in-depth follow-up studies for better understanding the role of plastic in the spread of antibiotic resistant pathogens in the marine environment are needed.

Supplementary Materials: The following are available online at http://www.mdpi.com/2076-2607/8/8/1200/s1, Figure S1: Phylogenetic tree for $c p h A \beta$-lactamases based on the amino acid sequence. The proteins highlighted with circles are new variants found in $A$. salmonicida isolates in this study. Accession numbers are presented for sequences downloaded from GenBank. PFM1 (QDC33502.1) representing subclass B2 metallo-beta-lactamase; PFM-1 from Pseudomonas fluorescens is used as an out group for construction of phylogenetic tree. Numbers at nodes are bootstrap values $(\%)$ based on 1000 resampled datasets; only values $>50 \%$ are given. Table S1: Identification and isolation source of the isolates obtained in the study. Table S2: Genome sequence assembly statistics for the whole genome sequences of the six isolates. Table S3: A list of virulence genes detected in the genome sequence of the isolates.

Author Contributions: N.P.M. conceived the study and designed the experiment. V.R. and P.S.N. performed the experiments and bioinformatic analysis with assistance from N.P.M., N.P.M. and V.R. analyzed the data. A.M.B. helped with plastic analysis. N.P.M. and V.R. drafted the manuscript. P.S.N. and A.M.B. provided critical inputs. N.P.M. acquired the project funding and was responsible for the overall direction of the project. All authors have read and agreed to the published version of the manuscript.

Funding: The study was funded by Institute of Marine research, under Ocean Health programme (project number-15495).

Acknowledgments: The authors acknowledge Didrik Hjertaker Grevskott from Institute of Marine Research (IMR) for help with sample collection. We acknowledge Ørjan Bjorøy from IMR for providing technical support and Julia Storesund from IMR for discussions regarding bioinformatics analysis. The authors also acknowledge Monica Sanden, Cecilie Smith Svanevik and Bjørn Tore Lunestad from IMR for supporting the work. The sequencing service was provided by the Norwegian Sequencing Centre (www.sequencing.uio.no), a national technology platform hosted by the University of Oslo and supported by the "Functional Genomics" and "Infrastructure" programs of the Research Council of Norway and the Southeastern Regional Health Authorities.

Conflicts of Interest: The authors have no conflict of interest.

Availability of Data: Genome sequences have been submitted to GenBank under the following genome accession numbers: JAACGC000000000, JAACGB000000000, JAACGA000000000, JAACFZ000000000, JAACFY000000000, JAACFX000000000 and JAACFW000000000. 


\section{References}

1. Shah, A.A.; Hasan, F.; Hameed, A.; Ahmed, S. Biological degradation of plastics: A comprehensive review. Biotechnol. Adv. 2008, 26, 246-265. [CrossRef]

2. Derraik, J.G. The pollution of the marine environment by plastic debris: A review. Mar. Pollut. Bull. 2002, 44, 842-852. [CrossRef]

3. Wilber, R.J. Plastic in the North Atlantic. Oceanus 1987, 30, 61-68.

4. Pruter, A.T. Sources, quantities and distribution of persistent plastics in the marine environment. Mar. Pollut. Bull. 1987, 18, 305-310. [CrossRef]

5. Yang, Y.; Liu, G.; Song, W.; Ye, C.; Lin, H.; Li, Z.; Liu, W. Plastics in the marine environment are reservoirs for antibiotic and metal resistance genes. Environ. Int. 2019, 123, 79-86. [CrossRef] [PubMed]

6. Blair, R.M.; Waldron, S.; Phoenix, V.; Gauchotte-Lindsay, C. Micro-and nanoplastic pollution of freshwater and wastewater treatment systems. Springer Sci. Rev. 2017, 5, 19-30. [CrossRef]

7. Koelmans, A.A.; Besseling, E.; Shim, W.J. Nanoplastics in the Aquatic Environment. Critical Review. In Marine Anthropogenic Litter; Springer: Cham, Switzerland, 2015; pp. 325-340.

8. Avio, C.G.; Gorbi, S.; Regoli, F. Plastics and microplastics in the oceans: From emerging pollutants to emerged threat. Mar. Environ. Res. 2017, 128, 2-11. [CrossRef] [PubMed]

9. Moore, C.J. Synthetic polymers in the marine environment: A rapidly increasing, long-term threat. Environ. Res. 2008, 108, 131-139. [CrossRef] [PubMed]

10. Gregory, M.R. Environmental implications of plastic debris in marine settings entanglement, ingestion, smothering, hangers-on, hitch-hiking and alien invasions. Philos. Trans. R. Soc. B 2009, 364, 2013-2025. [CrossRef]

11. Jepsen, E.M.; de Bruyn, P.J.N. Pinniped entanglement in oceanic plastic pollution: A global review. Mar. Pollut. Bull. 2019, 145, 295-305. [CrossRef]

12. Kühn, S.; Bravo Rebolledo, E.L.; van Franeker, J.A. Deleterious Effects of Litter on Marine Life. In Marine Anthropogenic Litter; Bergmann, M., Gutow, L., Klages, M., Eds.; Springer International Publishing: Cham, Switzerland, 2015; pp. 75-116. [CrossRef]

13. Kögel, T.; Bjorøy, Ø.; Toto, B.; Bienfait, A.M.; Sanden, M. Micro- and nanoplastic toxicity on aquatic life: Determining factors. Sci. Total Environ. 2020, 709, 136050. [CrossRef] [PubMed]

14. Li, W.C.; Tse, H.F.; Fok, L. Plastic waste in the marine environment: A review of sources, occurrence and effects. Sci. Total Environ. 2016, 566-567, 333-349. [CrossRef] [PubMed]

15. Zarfl, C.; Matthies, M. Are marine plastic particles transport vectors for organic pollutants to the Arctic? Mar. Pollut. Bull. 2010, 60, 1810-1814. [CrossRef] [PubMed]

16. Schneider, F.; Parsons, S.; Clift, S.; Stolte, A.; McManus, M.C. Collected marine litter-A growing waste challenge. Mar. Pollut. Bull. 2018, 128, 162-174. [CrossRef]

17. Bryant, J.A.; Clemente, T.M.; Viviani, D.A.; Fong, A.A.; Thomas, K.A.; Kemp, P.; Karl, D.M.; White, A.E.; DeLong, E.F. Diversity and activity of communities inhabiting plastic debris in the North Pacific Gyre. MSystems 2016, 1, e00024-16. [CrossRef]

18. Oberbeckmann, S.; Loeder, M.G.J.; Gerdts, G.; Osborn, A.M. Spatial and seasonal variation in diversity and structure of microbial biofilms on marine plastics in Northern European waters. FEMS Microbiol. Ecol. 2014, 90, 478-492. [CrossRef]

19. Eriksen, M.; Lebreton, L.C.; Carson, H.S.; Thiel, M.; Moore, C.J.; Borerro, J.C.; Galgani, F.; Ryan, P.G.; Reisser, J. Plastic pollution in the world's oceans: More than 5 trillion plastic pieces weighing over 250,000 tons afloat at sea. PLoS ONE 2014, 9, e111913. [CrossRef]

20. Arias-Andres, M.; Klümper, U.; Rojas-Jimenez, K.; Grossart, H.-P. Microplastic pollution increases gene exchange in aquatic ecosystems. Environ. Pollut. 2018, 237, 253-261. [CrossRef] [PubMed]

21. Booth, A.; Kubowicz, S.; Beegle-Krause, C.; Skancke, J.; Nordam, T.; Landsem, E.; Throne-Holst, M.; Jahren, S. Microplastic in Global and Norwegian Marine Environments: Distributions, Degradation Mechanisms and Transport. Miljødirektoratet M-918; SINTEF: Trondheim, Norway, 2017; pp. 1-147.

22. Jensen, S.; Lindbaek, M.; Gradmann, C.; Lie, A.K. Reflections about antibiotic resistance history in Norway. Erindr. Antibiot. Hist. Nor. 2010, 130, 2494. [CrossRef] 
23. Amalina, N.Z.; Santha, S.; Zulperi, D.; Amal, M.N.A.; Yusof, M.T.; Zamri-Saad, M.; Ina-Salwany, M.Y. Prevalence, antimicrobial susceptibility and plasmid profiling of Vibrio spp. isolated from cultured groupers in Peninsular Malaysia. BMC Microbiol. 2019, 19, 251. [CrossRef] [PubMed]

24. Baron, S.; Granier, S.A.; Larvor, E.; Jouy, E.; Cineux, M.; Wilhelm, A.; Gassilloud, B.; Le Bouquin, S.; Kempf, I.; Chauvin, C. Aeromonas diversity and antimicrobial susceptibility in freshwater-An attempt to set generic epidemiological cut-off values. Front. Microbiol. 2017, 8, 503. [CrossRef] [PubMed]

25. Bankevich, A.; Nurk, S.; Antipov, D.; Gurevich, A.A.; Dvorkin, M.; Kulikov, A.S.; Lesin, V.M.; Nikolenko, S.I.; Pham, S.; Prjibelski, A.D. SPAdes: A new genome assembly algorithm and its applications to single-cell sequencing. J. Comput. Biol. 2012, 19, 455-477. [CrossRef] [PubMed]

26. Tatusova, T.; DiCuccio, M.; Badretdin, A.; Chetvernin, V.; Ciufo, S.; Li, W. Prokaryotic Genome Annotation Pipeline. In The NCBI Handbook [Internet], 2nd ed.; Bethesda, US: Center for Biotechnology Information: Rockville, MD, USA, 2013; pp. 1-12.

27. Alcock, B.P.; Raphenya, A.R.; Lau, T.T.; Tsang, K.K.; Bouchard, M.; Edalatmand, A.; Huynh, W.; Nguyen, A.-L.V.; Cheng, A.A.; Liu, S. CARD 2020: Antibiotic resistome surveillance with the comprehensive antibiotic resistance database. Nucleic Acids Res. 2020, 48, D517-D525. [CrossRef] [PubMed]

28. Zankari, E.; Hasman, H.; Cosentino, S.; Vestergaard, M.; Rasmussen, S.; Lund, O.; Aarestrup, F.M.; Larsen, M.V. Identification of acquired antimicrobial resistance genes. J. Antimicrob. Chemother. 2012, 67, 2640-2644. [CrossRef] [PubMed]

29. Liu, B.; Zheng, D.; Jin, Q.; Chen, L.; Yang, J. VFDB 2019: A comparative pathogenomic platform with an interactive web interface. Nucleic Acids Res. 2019, 47, D687-D692. [CrossRef] [PubMed]

30. Larkin, M.A.; Blackshields, G.; Brown, N.P.; Chenna, R.; McGettigan, P.A.; McWilliam, H.; Valentin, F.; Wallace, I.M.; Wilm, A.; Lopez, R. Clustal W and Clustal X version 2.0. Bioinformatics 2007, 23, 2947-2948. [CrossRef]

31. Kumar, S.; Stecher, G.; Li, M.; Knyaz, C.; Tamura, K. MEGA X: Molecular evolutionary genetics analysis across computing platforms. Mol. Biol. Evol. 2018, 35, 1547-1549. [CrossRef]

32. Primpke, S.; Wirth, M.; Lorenz, C.; Gerdts, G. Reference database design for the automated analysis of microplastic samples based on Fourier transform infrared (FTIR) spectroscopy. Anal. Bioanal. Chem. 2018, 410, 5131-5141. [CrossRef]

33. Viršek, M.K.; Lovšin, M.N.; Koren, Š.; Kržan, A.; Peterlin, M. Microplastics as a vector for the transport of the bacterial fish pathogen species Aeromonas salmonicida. Mar. Pollut. Bull. 2017, 125, 301-309. [CrossRef]

34. Parker, J.L.; Shaw, J.G. Aeromonas spp. clinical microbiology and disease. J. Infect. 2011, 62, 109-118. [CrossRef]

35. Igbinosa, I.H.; Igumbor, E.U.; Aghdasi, F.; Tom, M.; Okoh, A.I. Emerging Aeromonas species infections and their significance in public health. Sci. World J. 2012, 2012, 1-13. [CrossRef]

36. Reith, M.E.; Singh, R.K.; Curtis, B.; Boyd, J.M.; Bouevitch, A.; Kimball, J.; Munholland, J.; Murphy, C.; Sarty, D.; Williams, J. The genome of Aeromonas salmonicida subsp. salmonicida A449: Insights into the evolution of a fish pathogen. BMC Genom. 2008, 9, 427. [CrossRef] [PubMed]

37. Austin, B.; Austin, D.A. Bacterial Fish Pathogens: Disease of Farmed and Wild Fish; Springer: London, UK, 1999.

38. Wiles, T.J.; Mulvey, M.A. The RTX pore-forming toxin $\alpha$-hemolysin of uropathogenic Escherichia coli: Progress and perspectives. Future Microbiol. 2013, 8, 73-84. [CrossRef]

39. Suarez, G.; Khajanchi, B.K.; Sierra, J.C.; Erova, T.E.; Sha, J.; Chopra, A.K. Actin cross-linking domain of Aeromonas hydrophila repeat in toxin A (RtxA) induces host cell rounding and apoptosis. Gene 2012, 506, 369-376. [CrossRef]

40. Bücker, R.; Krug, S.M.; Rosenthal, R.; Günzel, D.; Fromm, A.; Zeitz, M.; Chakraborty, T.; Fromm, M.; Epple, H.-J.; Schulzke, J.-D. Aerolysin from Aeromonas hydrophila Perturbs Tight Junction Integrity and Cell Lesion Repair in Intestinal Epithelial HT-29/B6 Cells. J. Infect. Dis. 2011, 204, 1283-1292. [CrossRef]

41. Rasmussen-Ivey, C.R.; Figueras, M.J.; McGarey, D.; Liles, M.R. Virulence Factors of Aeromonas hydrophila: In the Wake of Reclassification. Front. Microbiol. 2016, 7, 1337. [CrossRef] [PubMed]

42. Wedekind, J.E.; Trame, C.B.; Dorywalska, M.; Koehl, P.; Raschke, T.M.; McKee, M.; FitzGerald, D.; Collier, R.J.; McKay, D.B. Refined crystallographic structure of Pseudomonas aeruginosa exotoxin A and its implications for the molecular mechanism of toxicity. J. Mol. Biol. 2001, 314, 823-837. [CrossRef] [PubMed]

43. Li, G.; Howard, S.P. ExeA binds to peptidoglycan and forms a multimer for assembly of the type II secretion apparatus in Aeromonas hydrophila. Mol. Microbiol. 2010, 76, 772-781. [CrossRef] [PubMed] 
44. Schoenhofen, I.C.; Stratilo, C.; Howard, S.P. An ExeAB complex in the type II secretion pathway of Aeromonas hydrophila: Effect of ATP-binding cassette mutations on complex formation and function. Mol. Microbiol. 1998, 29, 1237-1247. [CrossRef]

45. Suarez, G.; Sierra, J.C.; Sha, J.; Wang, S.; Erova, T.E.; Fadl, A.A.; Foltz, S.M.; Horneman, A.J.; Chopra, A.K. Molecular characterization of a functional type VI secretion system from a clinical isolate of Aeromonas hydrophila. Microb. Pathog. 2008, 44, 344-361. [CrossRef]

46. Sha, J.; Rosenzweig, J.A.; Kozlova, E.V.; Wang, S.; Erova, T.E.; Kirtley, M.L.; van Lier, C.J.; Chopra, A.K. Evaluation of the roles played by Hcp and VgrG type 6 secretion system effectors in Aeromonas hydrophila SSU pathogenesis. Microbiology 2013, 159, 1120. [CrossRef] [PubMed]

47. Cattoir, V.; Poirel, L.; Mazel, D.; Soussy, C.-J.; Nordmann, P. Vibrio splendidus as the source of plasmid-mediated QnrS-like quinolone resistance determinants. Antimicrob. Agents Chemother. 2007, 51, 2650-2651. [CrossRef] [PubMed]

48. Poirel, L.; Rodriguez-Martinez, J.-M.; Mammeri, H.; Liard, A.; Nordmann, P. Origin of plasmid-mediated quinolone resistance determinant QnrA. Antimicrob. Agents Chemother. 2005, 49, 3523-3525. [CrossRef] [PubMed]

49. Potron, A.; Poirel, L.; Nordmann, P. Origin of OXA-181, an emerging carbapenem-hydrolyzing oxacillinase, as a chromosomal gene in Shewanella xiamenensis. Antimicrob. Agents Chemother. 2011, 55, 4405-4407. [CrossRef]

50. Ebmeyer, S.; Kristiansson, E.; Larsson, D.G.J. The mobile FOX AmpC beta-lactamases originated in Aeromonas allosaccharophila. Int. J. Antimicrob. Agents 2019, 54, 798-802. [CrossRef]

51. Ebmeyer, S.; Kristiansson, E.; Larsson, D.J. CMY-1/MOX-family AmpC $\beta$-lactamases MOX-1, MOX-2 and MOX-9 were mobilized independently from three aeromonas species. J. Antimicrob. Chemother. 2019, 74, 1202-1206. [CrossRef]

52. Dang, H.; Lovell, C.R. Microbial surface colonization and biofilm development in marine environments. Microbiol. Mol. Biol. Rev. 2016, 80, 91-138. [CrossRef]

53. Li, J.; Zhang, K.; Zhang, H. Adsorption of antibiotics on microplastics. Environ. Pollut. 2018, 237, 460-467. [CrossRef]

54. Huijbers, P.M.; Blaak, H.; de Jong, M.C.; Graat, E.A.; Vandenbroucke-Grauls, C.M.; de Roda Husman, A.M. Role of the environment in the transmission of antimicrobial resistance to humans: A review. Environ. Sci. Technol. 2015, 49, 11993-12004. [CrossRef]

55. Caruso, G. Microplastics as vectors of contaminants. Mar. Pollut. Bull. 2019, 146, 921-924. [CrossRef]

56. Jutkina, J.; Marathe, N.P.; Flach, C.F.; Larsson, D.G.J. Antibiotics and common antibacterial biocides stimulate horizontal transfer of resistance at low concentrations. Sci. Total Environ. 2018, 616-617, 172-178. [CrossRef] [PubMed]

57. Tucci, V.; Isenberg, H.D. Hospital cluster epidemic with Morganella morganii. J. Clin. Microbiol. 1981, 14, 563-566. [CrossRef] [PubMed]

58. Mastroianni, A.; Coronado, O.; Chiodo, F. Morganella morganii meningitis in a patient with AIDS. J. Infect. 1994, 29, 356-357. [CrossRef]

59. Liu, H.; Zhu, J.; Hu, Q.; Rao, X. Morganella morganii, a non-negligent opportunistic pathogen. Int. J. Infect. Dis. 2016, 50, 10-17. [CrossRef] [PubMed]

60. Visca, P.; Seifert, H.; Towner, K.J. Acinetobacter infection-An emerging threat to human health. IUBMB Life 2011, 63, 1048-1054. [CrossRef] [PubMed]

61. Hua, H.T.; Bollet, C.; Tercian, S.; Drancourt, M.; Raoult, D. Aeromonas popoffii urinary tract infection. J. Clin. Microbiol. 2004, 42, 5427-5428. [CrossRef]

62. Adeolu, M.; Alnajar, S.; Naushad, S.; Gupta, R.S. Genome-based phylogeny and taxonomy of the 'Enterobacteriales': Proposal for Enterobacterales ord. nov. divided into the families Enterobacteriaceae, Erwiniaceae fam. nov., Pectobacteriaceae fam. nov., Yersiniaceae fam. nov., Hafniaceae fam. nov., Morganellaceae fam. nov., and Budviciaceae fam. nov. Int. J. Syst. Evol. Microbiol. 2016, 66, 5575-5599.

63. Silva, M.M.; Maldonado, G.C.; Castro, R.O.; de Sá Felizardo, J.; Cardoso, R.P.; dos Anjos, R.M.; de Araújo, F.V. Dispersal of potentially pathogenic bacteria by plastic debris in Guanabara Bay, RJ, Brazil. Mar. Pollut. Bull. 2019, 141, 561-568. [CrossRef]

64. Stévant, P.; Rebours, C.; Chapman, A. Seaweed aquaculture in Norway: Recent industrial developments and future perspectives. Aquac. Int. 2017, 25, 1373-1390. [CrossRef] 
65. Fisheries, F. The State of World Fisheries and Aquaculture 2010; Food and Agriculture Organization of the United Nations: Rome, Italy, 2016.

66. Sentralbyrå, S. Akvakultur, 2018, Årlig Endelige Tall. Available online: https://www.ssb.no/jord-skog-jaktog-fiskeri/statistikker/fiskeoppdrett/aar (accessed on 5 May 2020).

67. FAO. Globefish highlights-A quarterly update on world seafood markets-April 2019 issues, with Jan.-Dec. 2018 statistics. Globefish Highlights 2019, 2, 1-68.

(c)

(C) 2020 by the authors. Licensee MDPI, Basel, Switzerland. This article is an open access article distributed under the terms and conditions of the Creative Commons Attribution (CC BY) license (http://creativecommons.org/licenses/by/4.0/). 